\title{
Challenge towards synthesis of non-silica-based hybrid mesoporous materials
}

\author{
-Level of compositional design and control of mesoporous materials achieved so far-
}

Tatsuo KIMURA

[Translation from Synthesiology, Vol.11, No.3, p.115-127 (2018)]

\begin{abstract}
Amphiphilic organic molecules have often been transformed into liquid-crystal structures in their concentrated solutions. This paper focuses on a group of porous materials, called "ordered mesoporous materials." Ordered mesoporous materials have nanostructures that replicate liquid-crystal structures. I report on the current level of compositional design that can be realized using mesoporous materials. In addition to silica-based materials, various inorganic compositions have been recently considered as possible alternatives. I have been striving to develop a more difficult method to obtain hybrid mesoporous materials in a non-silica-based system. To realize this, I have selected novel chemical resources for the synthesis of ordered mesoporous materials, proposed a new synthetic route, and realized reactivity control of such chemical resources and their functional design.
\end{abstract}

Keywords : Mesoporous structure, supramolecular template, compositional design, non-silica-based material, inorganic-organic hybrid framework

\section{Introduction}

Porous materials are materials that possess a large amount of void space within. Therefore, material surfaces are exposed in large amounts. This means that application is expected to various extended uses that utilize the characteristic of having an extremely large amount of surface area. The materials are categorized into microporous, mesoporous, and macroporous materials according to the pore size. According to the definitions of the International Union of Pure and Applied Chemistry (IUPAC), the microporous, mesoporous, and macroporous materials have pore size distributions in the ranges of $2 \mathrm{~nm}$ or less, $2-50 \mathrm{~nm}$, and $50 \mathrm{~nm}$ or more, respectively. A representative industrially important microporous material is zeolite (crystalline aluminosilicate) used in oil refinery processes and chemical product syntheses, and ion exchange zeolite is used as a catalyst for cleansing automobile exhaust gases. Silica gel used as a desiccant is the most famous mesoporous material, and this is also used as a filler in columns of analysis devices as an adsorption-separation agent.

Among the mesoporous materials, one must go back to around 1990 for the discovery of a silica porous body that was synthesized using a self-assembling characteristic of a surfactant (amphiphilic organic molecules). ${ }^{[1]}$ It drew worldwide interest due to the expectation for development of new uses for the structural characteristic unseen before in which uniform mesopores were arranged orderly in an extremely narrow pore size distribution. ${ }^{[2]}$ Although the image is a common sight now, one can imagine that the transmission electron microscope (TEM) photograph that showed the uniform ordered mesopores that was published in Nature at the time of its discovery made a great impression on materials researchers. ${ }^{[3]}$ Figure 1 (top) shows a typical analysis result of mesoporous silica. A nanoscale ordered structure can be seen from X-ray diffraction measurement in the low-angle range, and it is possible to directly visualize the structure through TEM observation. Also, by analyzing the shape of nitrogen adsorption isotherms, it is possible to calculate a specific surface area, pore capacity, and pore size distribution. However, impurities or materials that have not turned porous may be mixed. Therefore, to determine the success of mesoporous silica synthesis, it is necessary to see not only the uniformity and periodicity of mesopores, but also various analysis results comprehensively.

Figure 1 (bottom) is a summary of the examples of application development including the possibility of achieving mesoporosity through various material compositions. Normally, application development using functions of oxides is conducted. In cases of porous materials, development of applications as catalyst carriers and adsorbents is conducted to make use of pore space. In order to add functions that are not expressed with silica alone, functions (acidity, oxidation function, etc.) are added by introducing heteroelements, or organic groups are incorporated into the silica framework as in mesoporous organosilica (hybrid mesoporous material

Inorganic Functional Materials Research Institute, AIST 2266-98 Anagahora, Shimoshidami, Moriyama-ku, Nagoya 463-8560, Japan E-mail: t-kimura@aist.go.jp

Original manuscript received March 9, 2018, Revisions received June 1, 2018, Accepted June 6, 2018 
in which a silica framework, an inorganic species, and organic groups are compounded at molecular scale). In any case, the catalyst functions are designed by introducing components necessary for the achievement of functional expression. Since there is no other option than to use the pore environment provided by the final product, the design of the pore environment is important for the expressed function to efficiently progress. In this research, I present the difficulty of synthesis research and the level that I have currently achieved, taking as examples the mesoporous material composition design and the synthesis method of non-silica-based hybrid mesoporous materials for which control technologies have been advanced. Ultimately, if I utilize elemental technologies needed for functional design that were clarified in silica, it will be possible to design a chemical reaction field at nanoscale where an expressed function can progress efficiently as possible.

Figure 2 shows the types of surfactants generally used for the synthesis of mesoporous silica, the range of pore size control (1-100 nm or more), and comparison with a typical molecular size. In microporous materials, only small molecules such as benzene can be utilized. On the other hand, mesoporous materials can take in larger substrates into the pores. For example, there is rising expectation that the pores may be used as nanoscale synthesis vessels for fine chemicals or solidification media of huge functional molecules such as protein and enzymes (box of red dotted line). There are also possibilities of increasing performance that arise from increased molecular diffusion. As a guideline, the pore size range at which such functions are expressed effectively is shown (box of blue dotted line). Research of mesoporous materials progressed into a global research field due to the uniqueness of the material. Moreover, there was increased expectation that mesoporous materials might be synthesized by methods similar to silica but with non-silica compositions. ${ }^{[4]}$

If mesoporous organosilica precedingly developed from silica materials was from research activities "specializing on functional design," the primary research strategy of this research is "to design the pore environment." In general, hydroxyl groups are present on a silica surface, and it is said that some degree of hydrophilic property is seen on a flat surface. However, it is reported that hydrophobic behavior is seen in concave surfaces inside mesopores. Since mesoporous organosilica has hydrophobic organic groups within its framework exposed on mesopore surfaces, only application development assuming a hydrophobic pore environment can be conducted. Against this background, I set the initial goal of this research as "designing a hydrophilic surface structure" that was never observed in other mesoporous materials. Mesoporous aluminophosphate has extremely low structural stability and was not suitable for application development. However, it was reported to be the only mesoporous material with a hydrophilic surface structure, ${ }^{[5]}$ and I decided to propose a design guideline for a surface structure using this finding as a hint.

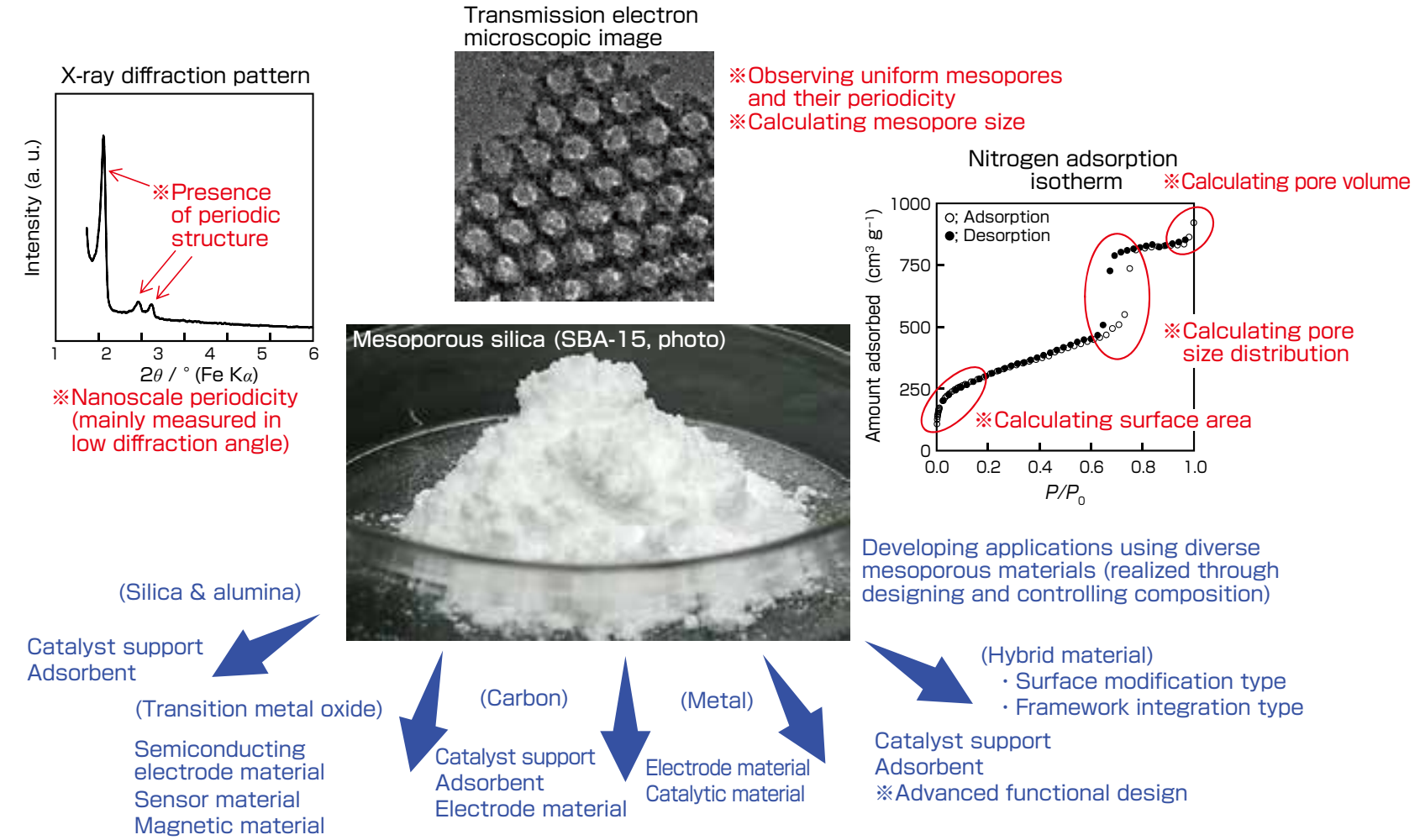

Fig. 1 Representative analyses of mesoporous silica and examples of developing applications using diverse mesoporous materials 


\section{Summary of extension of compositional control range for mesoporous materials and technological issues}

In this study, I discuss raising the level of control technology for framework composition of mesoporous materials. From the viewpoint of the synthesis chemistry, I summarize the difficulty of achieving mesoporosity in non-silica-based materials, the technological issues that prevent inorganicorganic compounding, while comparing with hybridization (inorganic-organic compounding) of the silica materials. The main elemental technologies of synthetic research for mesoporous materials are as follows:

(1) Selection of appropriate inorganic starting material, and reaction control of inorganic species in solution,

(2) Design of interaction between inorganic species and amphiphilic organic molecules in solution,

(3) Understanding the self-assembly behaviors of amphiphilic molecules that are newly produced by interaction,

(4) Adjustment of the process of liquid-crystal-like structure formation and the following polymerization of inorganic species,

(5) Development of removal method of amphiphilic organic molecules, and

(6) Process design assuming application development in thin films, powders, and others.

Figure 3 shows these elemental technologies in conjunction with each stage of formation of mesoporous silica precursors. The most important point here is to understand all elemental technologies comprehensively. That is, this research result, or the composition design technology of advanced mesoporous materials, could not have been made unless these elemental technologies were integrated.

The formation mechanism of mesoporous silica precursors that was shown immediately after the discovery of mesoporous silica drew controversy. If one understood that the precursors were formed by the liquid crystal template route as shown in Fig. 3 (top), it was sufficient to believe that space design at nanometer level could be done easily inside oxide materials. However, as a result of follow-up investigation, it was found that in most cases, the precursors (composite of liquid-crystal-like structured silica and amphiphilic organic molecules) are produced by concerted organization, as shown in Fig. 3 (bottom), rather than the liquid crystal template route. First, the hydrophilic regions of amphiphilic organic molecules interact with the dissolved silicate species in oligomer form. At this point, if the molecular size of inorganic species is too big, precursors with structural order cannot be obtained, or precipitates are formed beforehand. If interaction with inorganic species goes well, it can be considered that new inorganic-organic composite molecules with amphiphilic properties have been formed. If self-assembly of this composite molecule and the bonding of inorganic species occur simultaneously (concertedly), precursors with high structural order containing liquid crystal structures are obtained. Finally, when organic molecules are removed by firing or other methods so the structural order is not broken, an orderly mesospace is formed inside the material.

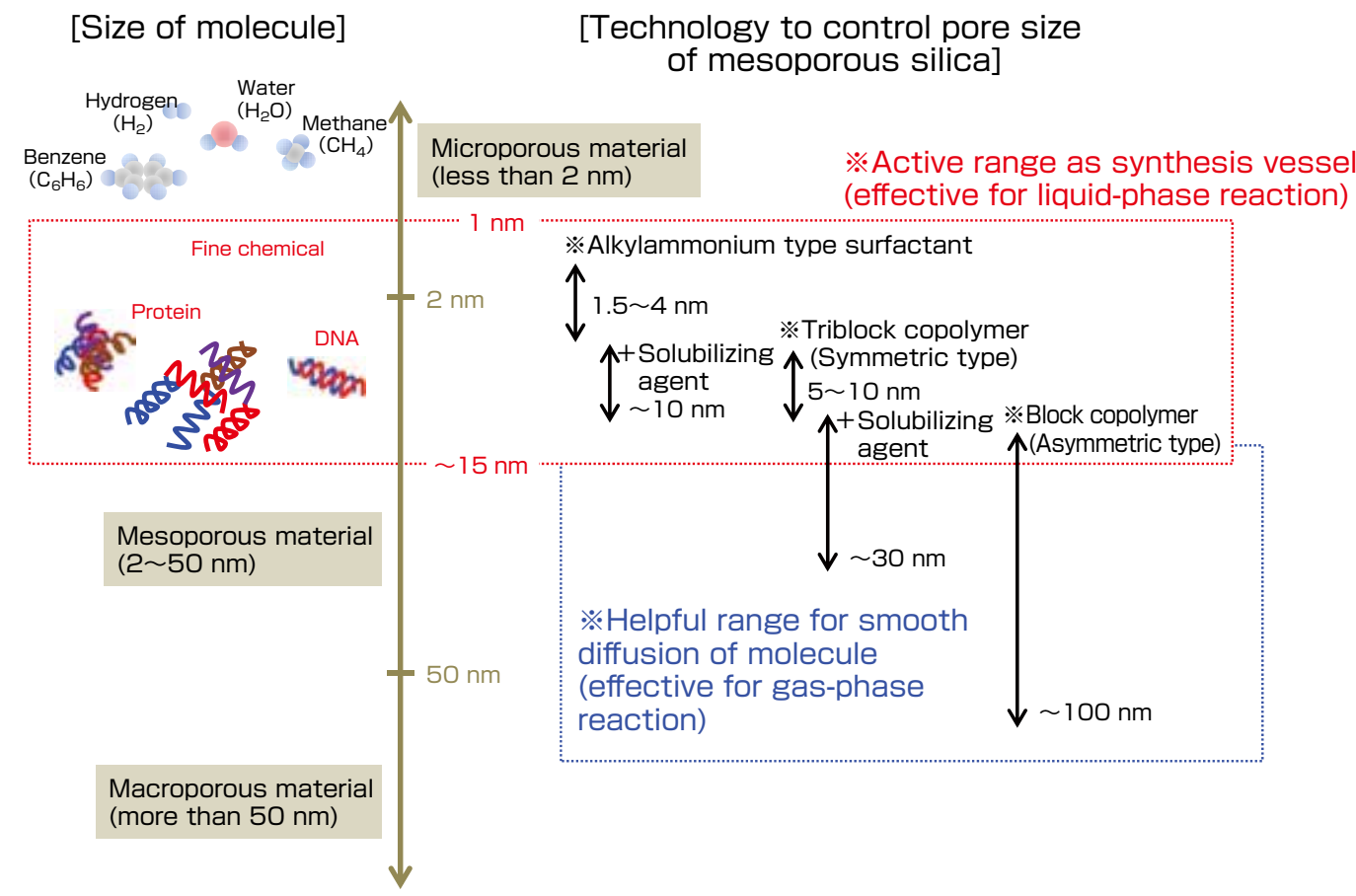

Fig. 2 Comparison between molecule size and range of pore size control of mesoporous silica 


\subsection{From silica to its hybrid (organosilica)}

For cases in which synthesis is started from silica starting materials such as tetra-alkoxysilane, there are sufficient findings in silicate chemistry. ${ }^{[6]}$ This means that the synthesis is done in an environment in which hydrolysis and polycondensation reactions can be controlled easily in solution. Moreover, because basic scientific understanding that conditions and reaction processes of dissolved silicate species can be traced by ${ }^{29} \mathrm{Si}$ NMR has grown, it is actually possible to synthesize diverse types of mesoporous silica. Inorganic-organic compounding of the framework in silica materials can be relatively easily achieved, even by using silane compounds bridged with organic groups as starter materials. ${ }^{[7]}$ Although reactivity of silane compounds may change, findings of silicate chemistry can be used fully, such as synthesizing by selecting either acidic or alkaline conditions. As shown in Fig. 4, there are reports of mesoporous organosilica synthesis from silane compounds bridged by various organic groups. Also, inorganic and organic groups are arranged alternately at molecular scale, and intermolecular interaction between organic groups within silane compounds may give periodicity to the arrangement of the organic and inorganic species. ${ }^{[8][9]}$ Simple organic groups are only expected to have the role of merely making pore surfaces hydrophobic. On the other hand, there is work done on utilizing the functionality or designability of organic groups themselves. For example, technology to capture light energy is being studied by forming a metal complex using bipyridine arranged as footholds on framework surfaces. ${ }^{[10]}$

\subsection{From silica to non-silica: Difficulty to synthesize}

\section{non-silica-based mesoporous materials}

The knowledge of silicate chemistry does not apply at all to the synthesis of non-silica-based mesoporous materials. Here, I shall explain using oxides such as alumina and titania as representatives of non-silica-based materials. Non-silicabased inorganic starters have severe reactions in solution. Therefore, chemical modifiers are used or non-aqueous systems are applied for reactions, and various other measures are reported as control methods to delay the general solgel reaction. However, combining such reaction control technology to the concerted organization route (Fig. 3) to obtain precursors for mesoporous materials has not been done fully. Since efforts are only mainly applied to the initial stage of the reaction, subsequent bond formation among inorganic species cannot be controlled. Therefore, self-assembly and framework formation are not adjusted to an appropriate speed, and inorganic materials are precipitated without being fully incorporated into amphiphilic organic molecules. Such behavior can be understood as follows: strength of bond energy (covalent bond) of inorganic framework $\gg$ energy of interaction (electrostatic interaction, hydrogen bond, etc.) of inorganic species and amphiphilic organic molecule $>$ power of energy of self-assembly. To obtain a mesoporous structure with high structural order, how to control bond formation of inorganic species in solution is the most important component.

For non-silica-based materials for which reactions are difficult to control, precision is required to control the reaction in solution. In other words, the fact that most reports for alumina and titania are limited to films shows

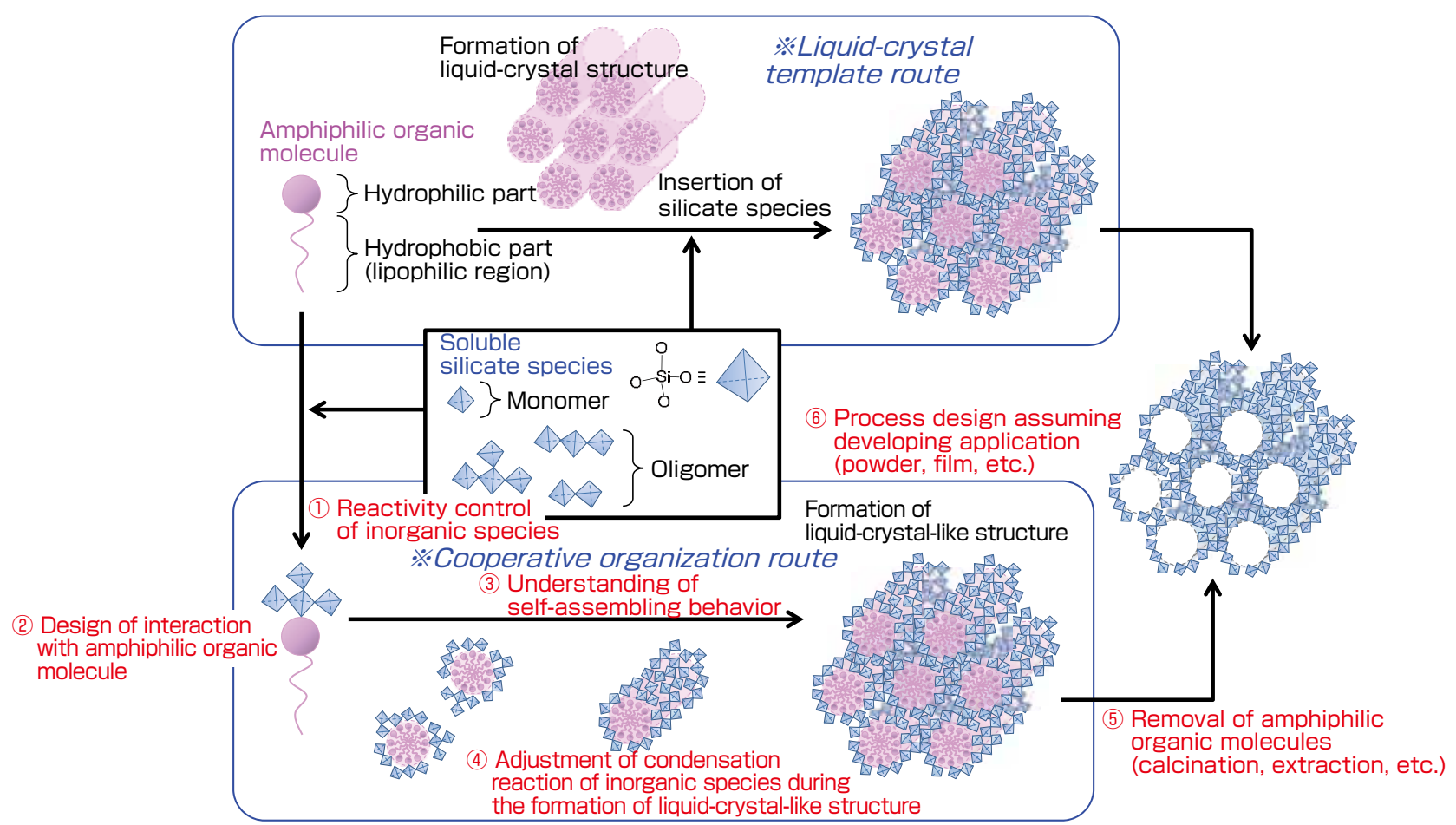

Fig. 3 Formation mechanism of mesoporous silica: Liquid-crystal templating and cooperative organization routes 
the difficulty of obtaining mesoporous material from nonsilica-based materials. To prevent formation of precipitation while controlling bond formation of inorganic species, first, a transparent precursor solution is prepared. Next, a solvent evaporation method ${ }^{[11][12]}$ is used to rapidly form a mesoporous structure to obtain a highly ordered mesoporous film. Preparation of a precursor solution is done mainly with an ethanol solvent. However, an alkoxide starting material cannot interact with the hydrophobic region of amphiphilic organic molecules. To slowly promote progression of hydrolysis and condensation reactions of alkoxide materials, a small amount of hydrochloric acid is added. Even then, when amphiphilic organic molecules and dissolved inorganic species interact, solvent evaporation is immediately promoted by processes such as spin coat and spray-dry, to bring them to a quick finish as precursors of mesoporous materials. ${ }^{[13]}$ Even more troublesome is the fact that polymerization reaction of oxide materials progresses even after film formation. As a desperate measure to forcibly delay this reaction, sometimes, the product may be placed in a freezer (about $-20^{\circ} \mathrm{C}$ ).

In the actual follow-up test, it has been confirmed that the structural order greatly decreases if it is not placed in a freezer. This is fine for goal-oriented basic research of performance evaluation of materials or for studying structure correlation. However, this cannot lead to process design for mass synthesis or mass production for practical use. Moreover, surprisingly, a majority of non-silicabased mesoporous materials presented as powder samples in published papers are actually synthesized by a solvent evaporation method. I have heard that there are cases in which the precursor solution is spread thinly as possible and then samples are collected by film formation. As a result of the difficulty of synthesizing non-silica-based mesoporous materials, such heavy-handed synthesis methods are used worldwide. Synthesis research mindful of process design suitable for practical application cannot be done unless understanding is deepened for the reactivity of inorganic materials and composition control technology is advanced in a true sense. In addition, there are expectations for the material to be used as a catalyst carrier by only using the effect of increased surface area like alumina. In most cases, sufficient function expression cannot be expected unless the crystalline property of the oxide framework is increased. For those that realized the synthesis of mesoporous film using oxides of transition metal, proposals were made for new application technologies as device parts such as sensor materials or electrode materials. Therefore, I did see effects of increasing adsorption volume of photo-responsive molecules or adsorption sites of sensing target components. However, since it was not possible to increase the crystalline property sufficiently while maintaining porosity derived from the mesoporous structure, it seemed that the effect of achieving mesoporosity was limited.

\section{Non-silica-based hybrid mesoporous material: Toward building of substance group}

As discussed above, functional design derived from silica cannot be expected. In starting this research, it was necessary to simultaneously realize inorganic-organic compounding of the framework, in addition to achieving mesoporosity in nonsilica-based materials. I set opening the way to this advanced material design as the ultimate goal of synthesis study. When I started, in reality, there was not even a way of synthesizing non-silica-based hybrid mesoporous materials. If I could develop a universal synthesis technology for a mesoporous material that was non-silica-based and was composed of an inorganic-organic framework, I could clear the way toward advanced material design, such as arbitrarily changing the environment inside the nanospace from hydrophobic to hydrophilic using properties of non-silica material surfaces,

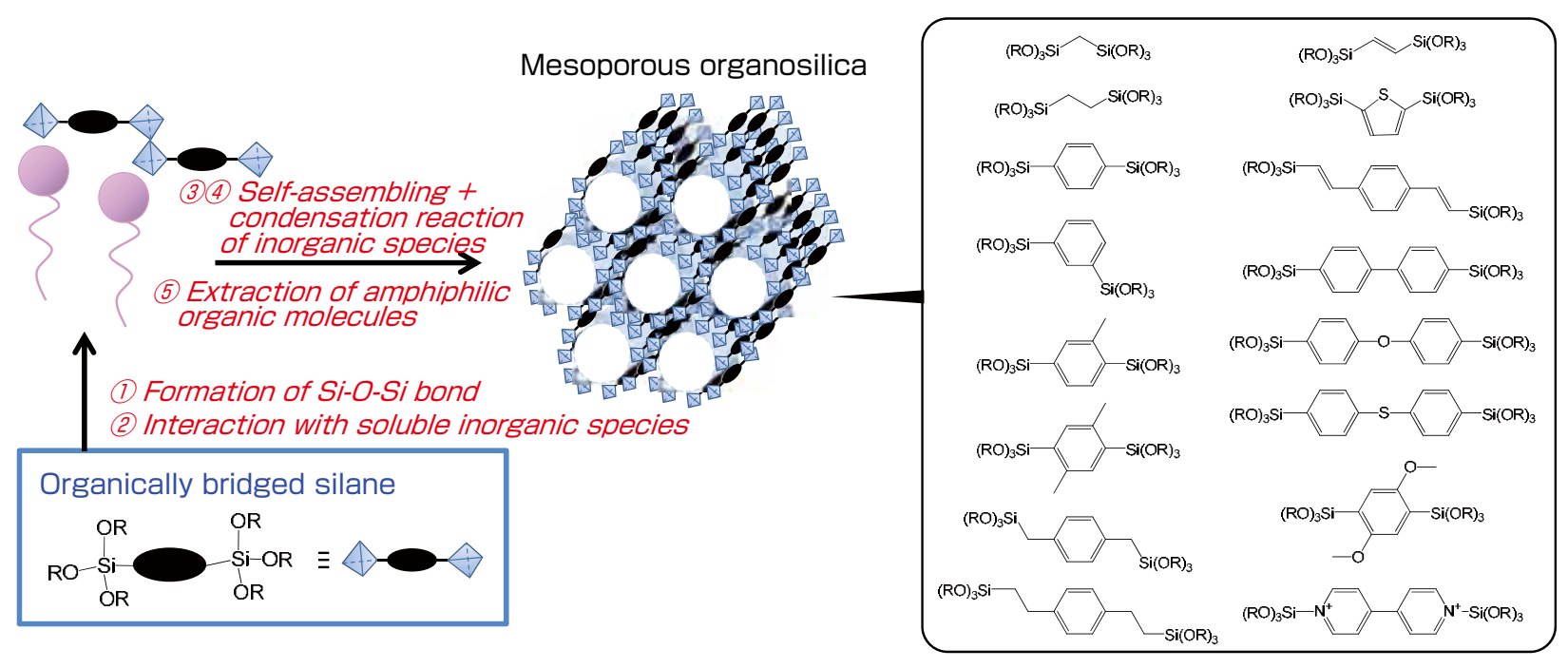

Fig. 4 Synthesis of mesoporous organosilica: Variation of organic groups (with the number of significant technologies shown in Fig. 3) 
or compounding with functions derived from inorganic species. As mentioned earlier, the progress from silica to organosilica went relatively smoothly. On the other hand, why did not the research for non-silica oxides lead to synthesis research for inorganic-organic compounding? It was probably because rather than the difficulty in controlling the reaction of a starting material, there was no such starting material. Other than silica, alkoxide materials or similar compounds that include bridged organic groups in the structure are not available commercially. In reality their synthesis methods are not reported. I found three types of tin compounds that were bridged by organic groups. ${ }^{[14]}$ If I had to develop synthesis methods of such compounds for each metal species, I would have to spend an enormous amount of energy on preparing raw materials for mesoporous materials, and I thought this was not a realistic approach.

\subsection{Proposal of synthesis method: Limit of organically bridged phosphonic acid}

From the above background, I have searched for compounds that are capable of bond formation with various metal species and included bridged organic groups within the molecular structure. As a result, I proposed the use of bisphosphonate compounds (similar to phosphoric acid that are bridged with organic groups). The synthesis technology for phosphonic acid was almost established. Moreover, in the synthesis of metal phosphates, for example, if the mol of phosphoric acid in solution was the same as the metal species, the reaction of the metal species would be inhibited, and I thought this advantage could be utilized. The mesoporous material was synthesized using commercially available phosphonates bridged with organic groups. Since I set the design of a "hydrophilic surface structure" as the first research goal, initially I spent my efforts on the synthesis of mesoporous aluminum phosphonates in which aluminum was the metal species. As a result, I was able to propose a synthesis route of a non-silica-based hybrid mesoporous material, as shown in Fig. 5 for the first time. ${ }^{[15]}$ For verification, I selected the simplest reaction of methylene bridged phosphonates and aluminium isopropoxide that is the aluminum source, and conducted the synthesis using an alkyltrimethylammonium ( $\mathrm{C}_{n}$ TMA) surfactant under alkaline conditions. The facts that the structural order could not be obtained sufficiently when achieving mesoporosity and that low-temperature firing had to be used since the surfactant could not be extracted (the bond between phosphorus atoms and bridged organic groups is partially disconnected) were seen as new issues.

The first issue, to improve the orderliness of the mesoporous structure, was not too difficult. ${ }^{[16]}$ The structural order improved greatly just by using methylene bridged phosphonates and aluminum chloride $\left(\mathrm{AlCl}_{3}\right)$ with suitable reactivity as the aluminum source, and by changing the synthetic condition to an acid condition. As a surfactant,

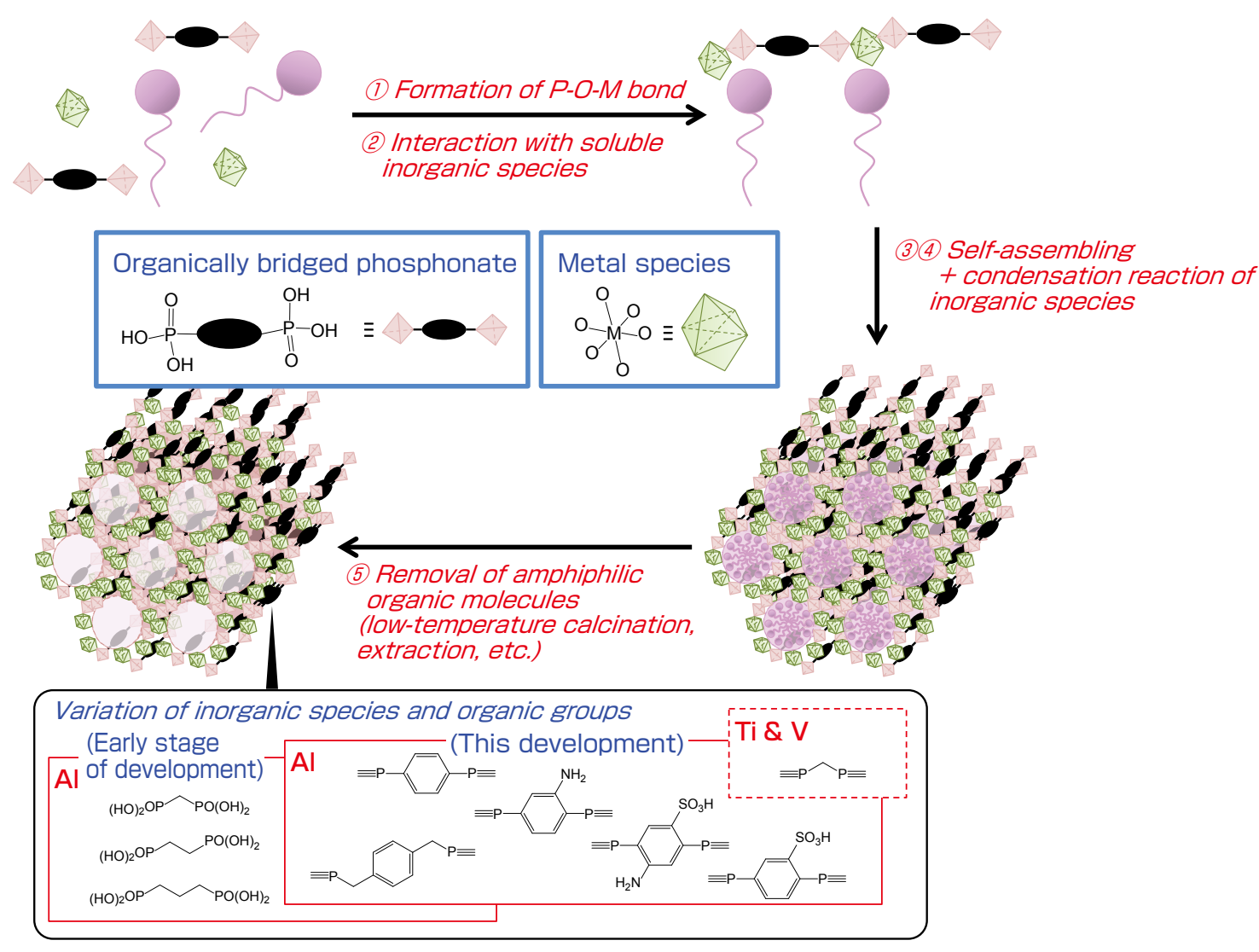

Fig. 5 Proposal of the synthesis route from organically bridged phosphonate compound, and progress in composition control technology (with the number of significant technologies shown in Fig. 3) 
not only $\mathrm{C}_{n} \mathrm{TMA}$, but also alkyl polyoxyether $\left(\mathrm{C}_{n} \mathrm{EO}_{m}\right)$ or polyoxyethylene-polyoxypropylene-polyoxyethylene triblock copolymer $\left(\mathrm{EO}_{n} \mathrm{PO}_{m} \mathrm{EO}_{n}\right)$ could be used. ${ }^{[16][17]}$ By using the changes in alkyl chain length and different polymerization number, it was confirmed that the pore size could be controlled in the range from around $2 \mathrm{~nm}$ to little less than $10 \mathrm{~nm}$. At this stage, the surfactant was removed by low-temperature (for example $400{ }^{\circ} \mathrm{C}$ ) firing, and therefore, I could only make products with bridged organic groups with relatively high heat resistance, such as methylene groups or ethylene groups. Using a similar synthesis method, for example, I attempted synthesis from benzene bridged phosphonates that could be functionally designed and had high heat resistance. However, I was unable to obtain mesoporous aluminum phosphonates with high structural order. The details will be explained later, but this presented the limit of synthesizing from organically bridged phosphonates. That is, I reached the conclusion that it was difficult to extend this substance unless there was a breakthrough in the precision reactivity control of the starting material.

To solve the second issue, the development of an extraction method of the surfactant, efforts were made by trial and error. As a result, it was found that $\mathrm{C}_{n} \mathrm{EO}_{m}$ and $\mathrm{EO}_{n} \mathrm{PO}_{m} \mathrm{EO}_{n}$ could be dissolved (removed) simply by heating in an acetone solvent. ${ }^{[18][19]}$ I achieved a situation in which a hybrid framework could be designed without considering heat resistance of bridged organic groups, and this was an extremely important result that pushed the research forward. It is thought that the phosphate $(\mathrm{P}-\mathrm{OH})$ group on the solid surface showing acidity acted as a catalyst to break down the EO and PO units. In mesoporous aluminophosphate, the stability of the mesoporous structure was so low that the structural order gradually degenerated even when there was steam present. Therefore, in a case of aluminum phosphonates that contained an aluminophosphate-like framework, it was important to do acetone treatment so there would be few water $\left(\mathrm{H}_{2} \mathrm{O}\right)$ molecules as possible. It was thought impossible to conduct performance evaluation for mesospace with hydrophilic environment unless this low stability was resolved. Fortunately, it was found that the stability of a mesoporous structure increased with the introduction of bridged organic groups. As a hydrophobic organic group was introduced proximal to the aluminophosphate-like framework, hydrolysis by $\mathrm{H}_{2} \mathrm{O}$ molecules was inhibited to some degree. As a result of introducing an organic group, the material surface became somewhat hydrophobic. Understanding this as being a local structure, the aluminophosphate-like structure that is the point of absorption of $\mathrm{H}_{2} \mathrm{O}$ molecules will remain exposed at the pore surface. That is, the road to performance evaluation of a hydrophilic surface environment or its proximity was not closed.

\subsection{Reactivity control of starting material: Possibility of phosphonate compounds}

The reactivity of bisphosphonate is changed dramatically when organic groups with high electron density such as benzene linkers are bridged. In this case, the reactivity with $\mathrm{AlCl}_{3}$ was decreased, and it was thought that mesoporous materials could not be synthesized. ${ }^{[20]}$ Here, synthesis was done using a mixed solvent of ethanol-water, but it was necessary to consider the solubility of organically bridged phosphonates in the solvent. For example, xylene bridged phosphonate was not dissolved in an ethanol-water mixed solvent, and even preparation of a precursor solution could not be done. In synthesis from bisphosphonates bridged by simple alkyl groups, mesoporous materials could be synthesized in an optimal synthetic condition even if organic groups were slightly different. When the property changed greatly as in benzene linker, the optimal synthetic condition could no longer be applied. For example, in the synthesis of mesoporous metal phosphate, the importance of appropriately selecting the difference between acid and alkaline of the starting material has been reported. ${ }^{[21]}$ In accordance with reactivity in the early reaction, in this synthesis system, when bisphosphonate ester $\left(\mathrm{H}_{5} \mathrm{C}_{2} \mathrm{O}\right)_{2} \mathrm{OP}-\mathrm{R}-\mathrm{PO}\left(\mathrm{OC}_{2} \mathrm{H}_{5}\right)_{2}$ was used as a starting material instead of its acid form $(\mathrm{HO})_{2} \mathrm{OP}-$ $\mathrm{R}-\mathrm{PO}(\mathrm{OH})_{2}(\mathrm{R}=$ bridged organic group), its reactivity with $\mathrm{AlCl}_{3}$ was too high and a gel was formed. Considering that the reactivity of phosphonates was insufficient, it was noted that reactivity with $\mathrm{AlCl}_{3}$ could be designed appropriately if there was a similar compound with reactivity somewhere in between that of phosphonates and esters.

\subsection{Diversification of organic groups: From alkyl group to aromatic compounds}

Though it is referred to as a phosphonate compound here, phosphonic acid is made by treating phosphonate esters in excessive amount of aqueous hydrochloric acid solution. If the treatment is done in conditions in which hydrochloric acid is lacking against the number of ester groups, a compound in an intermediate condition, that is, a phosphonate compound in which acid and esters coexist in the same molecular structure is obtained. I thought it would be possible to conduct continuous reaction control if the percentage was changed arbitrary. Here, the actual synthesis of mesoporous aluminum phosphonates from benzene bridged phosphonate esters when $\mathrm{AlCl}_{3}$ was used as the aluminum source will be explained. Synthesis was done using as starting material phosphonate compounds with different degrees of hydrochloride treatment on bisphosphonate esters. As a result I succeeded in obtaining a mesoporous film with extremely high structural order. ${ }^{[22]}$ The result of TEM observation is shown in Fig. 6. It can be seen that the mesopores are arranged evenly throughout the film.

I also obtained a mesoporous film using a similar approach from phosphonate compounds bridged by aromatic 
compounds such as xylene linkers. Moreover, I worked on increasing my skills to synthesize phosphonate esters in which the molecular structure was arbitrarily designed. Through such efforts, I achieved synthesis of mesoporous films from organically bridged phosphonate compounds in which amino groups $\left(-\mathrm{NH}_{2}\right)$ or sulfonic acid groups $\left(-\mathrm{SO}_{3} \mathrm{H}\right)$ were added to the bridged benzene ring. Figure 5 is a summary of compounds that were introduced as bridged organic groups. Not only simple organic groups, but also various aromatic compounds that could be functionally designed were introduced. This indicates that composition design technology has advanced to the level in which application research could be conducted as in silica. Therefore, the design of a "hydrophilic surface structure" and "survey of its specificity" for the "design of the pore environment" that are primary research strategies of this research have been mostly realized.

\subsection{Diversification of inorganic composition: From aluminum to transition metals}

Up to this point, the synthesis research that centered on aluminum as metal species was described. As the next step, I worked on the synthesis with metal species other than aluminum and started preliminary experiment for "controlling the pore environment." If diversification of inorganic species could be realized, it would be possible to use properties of inorganic unit surfaces that is traceable to the types of inorganic species. For example, in aluminum, since $\mathrm{H}_{2} \mathrm{O}$ molecules can be coordinated until tetracoordinate aluminum species $\left(\mathrm{AlO}_{4}\right)$ become 6-coordinate species $\left(\mathrm{AlO}_{6}\right)$ as a property of an aluminophosphonate-like inorganic unit, the surface behaves in a hydrophilic manner. Therefore, if synthesis could be done by other metal species, changes will be seen in the $\mathrm{H}_{2} \mathrm{O}$ adsorption behavior since those metal species would not become tetracoordinates.
Here, as the simplest example, the result is shown for synthesizing a mesoporous film of metal phosphonates from phosphonate compounds in which methylene bridged phosphonate esters are partially acid treated. It was confirmed that a mesoporous film could be obtained when titanium chloride $\left(\mathrm{TiCl}_{4}\right)$ was used as the inorganic material, as well as vanadium chloride $\left(\mathrm{VCl}_{3}\right)$ that does not have high reactivity. However, since $\mathrm{TiCl}_{4}$ has extremely high reactivity, the precursor solution was prepared using non-aqueous solution (ethanol). It is thought difficult to form a framework (formation of M-O-P bond) from metal species (M) that is present as ion species in solution. If the metal species (M) is capable of covalent bonding through phosphorus $(\mathrm{P})$ and oxygen $(\mathrm{O})$ atoms, I believe a mesoporous material with a new composition can be designed by understanding and controlling the reactivity of those metal species in solution.

\section{Process design assuming practical application using mesoporous material}

In this paper, the major elemental technologies for synthesis research of mesoporous materials are categorized from (1) to (6). For example, as shown in Fig. 5, it was explained that I was able to synthesize a hybrid mesoporous material using non-silica as a result of integrating (1) to (5). I present my conclusion by providing a discussion of the final elemental technology "(6) Process design assuming application development." Normally, materials development starts from fulfilling the demand for high performance against "social demand (application development)" as shown in Fig. 7 (top). If it is a replacement of an existing material (improvement research), the usage is already predetermined. Therefore, if high performance can be achieved through a similar synthesis system, the level of performance improvement that can be achieved is confirmed. If there are no issues about the cost of raw materials or manufacturing processes, it

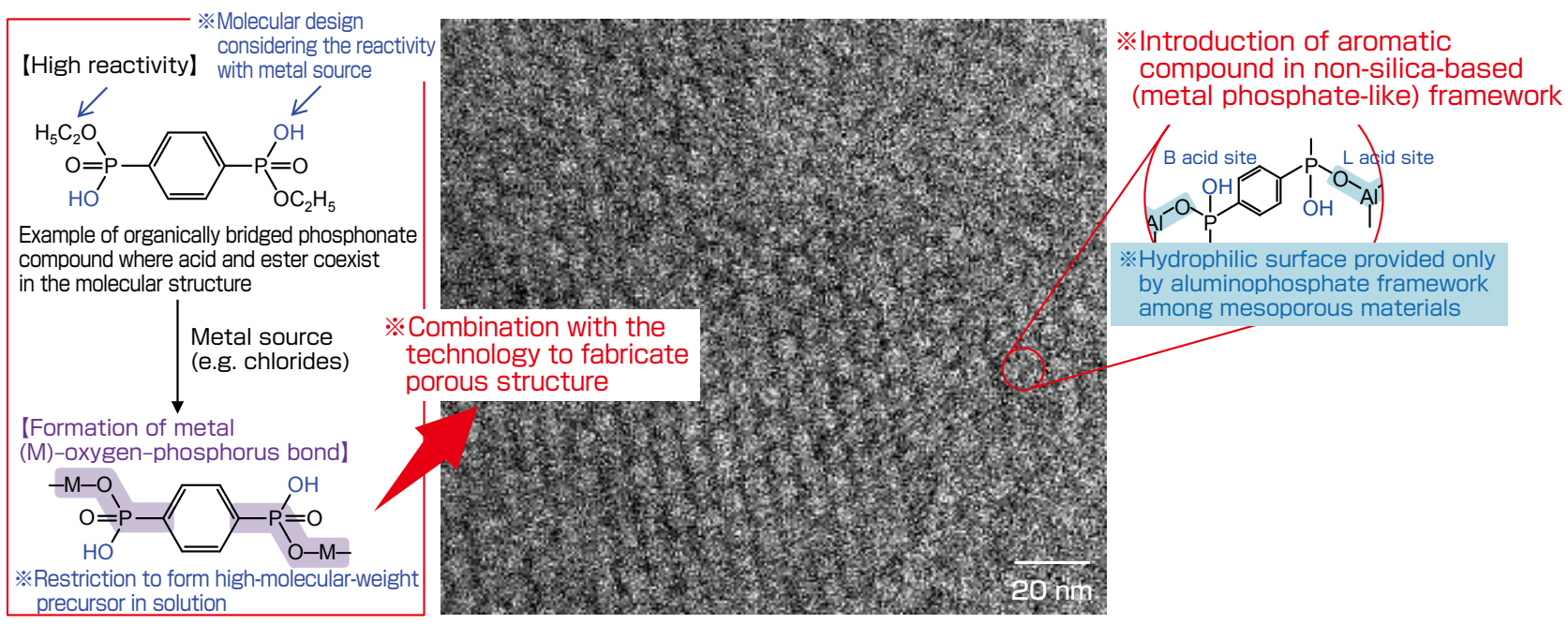

Fig. 6 Reaction of benzene bridged phosphonate compound and metal source, which combined with the technology to fabricate mesoporous structure 
will become a candidate alternative material. On the other hand, in cases that start with a discovery of a new material (dramatically enhanced function), there is a possibility of technological innovation. However, I all know that there are plenty of technological hurdles that must be overcome before practical application. Depending on how the new material is to be used, even the synthesis method that was first found may have to be altered. In general, this corresponds to the development of control technology for "composition," "structure," and "form" in synthesis research.

In a case of materials development in which mesoporous technology is used, materials synthesis is attempted by clarifying the developmental factors that are to be targeted. This is explained in Fig. 7 (bottom). If the performance in demand is clear, some hints may be obtained for candidate composition to achieve that function through surveys of existing research. Limited to the cases in which performance improvement can be expected through structure control (in this research, mainly achieving mesoporosity), I start the development of porosity technology using materials with candidate composition. If the form in which it will be used is set, the process of sample preparation as a film or powder can be determined. For example, say that one prepares a homogenous precursor solution, particularly a transparent one. In this case, separation of films and powder is possible only by coating or spray-drying, as the process used for both is solvent evaporation by which precursors for mesoporous materials is made. Therefore, development of structural control and form control can be construed as development of elemental technologies that can be conducted together. In the sense that formation of mesoposority is conducted for selected candidate composition, the composition is not the target of control. In synthesis research for mesoporous materials like this research, it is most important to properly understand the reactivity of raw materials in solution to advance mesoporosity technology.

There is another important point in achieving assumed performances to the maximum. In most cases, the precursor materials of mesoporous materials are obtained with inorganic frameworks of amorphous conditions, and it is necessary to crystallize the inorganic frameworks after achieving mesoporosity, if the expected performance is derived from the functional expression of crystalline materials. Since the inorganic framework can be crystallized by heat treatment (firing), additional process is not necessary. As mentioned before, if crystallization goes too far, the mesoporous structure disintegrates in most cases. Therefore, maximizing the crystallized ingredient without losing the effect of mesoporosity will be the aim of material design. Also, ones with larger pore sizes tend to have mesoporous structures that do not disintegrate even with crystallization. In such cases, the merit of "improved dispersibility" is obtained as well as "increased crystallization." Therefore, it is important to develop a precise synthesis technology by which the performance is maximized through the effects of surface area, crystallization, and dispersibility.

\section{Future issues and prospects}

The synthesis technology for "non-silica-based hybrid mesoporous materials" that was developed in this research showed major progress and has potential for greatly expanding the compositional control range of ordered mesoporous materials. However, mesoporous aluminum phosphonates, which were synthesized using phosphonic acid bridged with an alkyl group at the start of the research, was collected as powder. ${ }^{[15]-[19]}$ On the other hand, formation of films was the main theme of research for phosphonate compounds bridged with various aromatic compounds that were recently successfully developed. ${ }^{[22]}$ Fortunately, powder
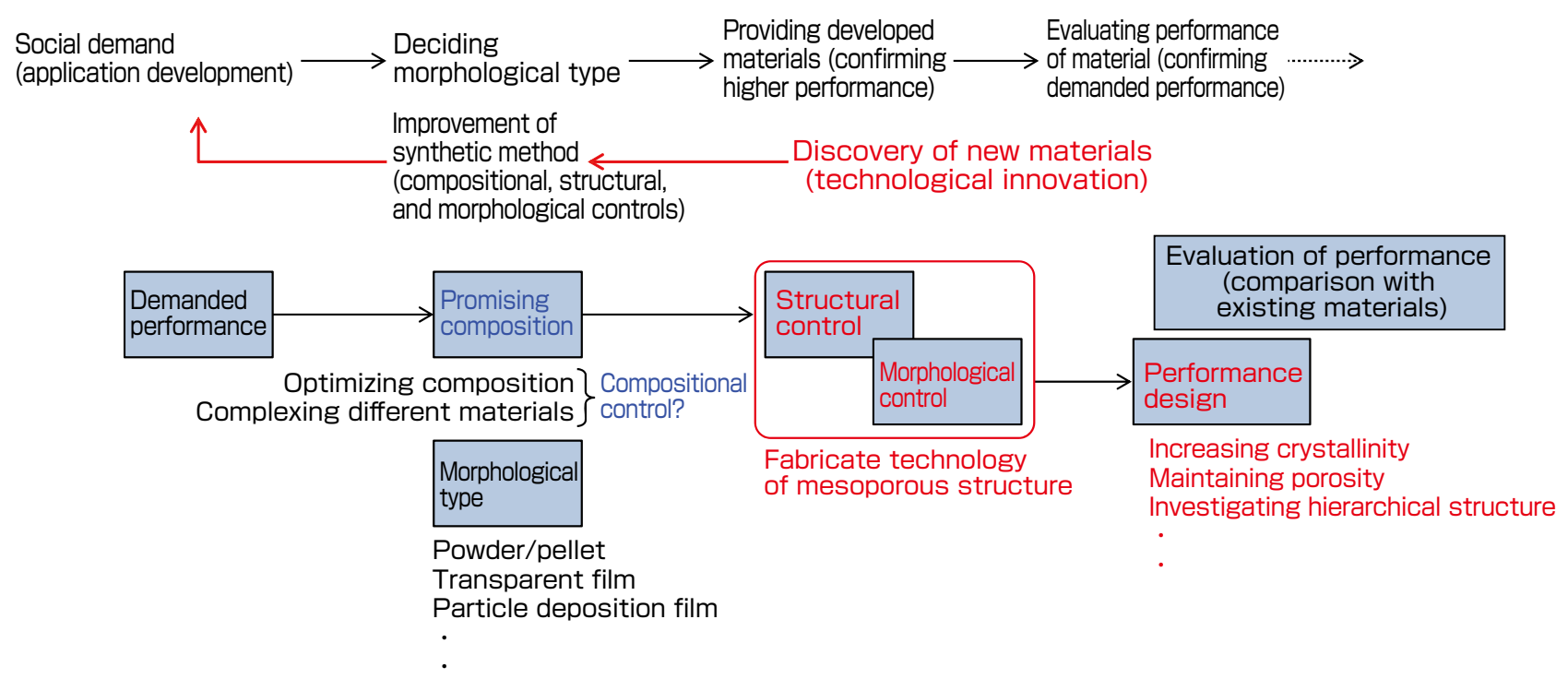

Fig. 7 Basic guideline for the materials design to develop applications 
synthesis of mesoporous aluminum phosphonates was researched using a spray-drying process that is known as a synthesis method including a solvent evaporation process similar to film synthesis, ${ }^{[23][24]}$ and the understanding of this process was advanced. ${ }^{[25]}$ Therefore, according to the demand of application development, it will become possible to provide various mesoporous metal phosphonates that were designed recently or will be designed in the future, as powder samples.

The "reactivity control of phosphonate compounds" was a breakthrough to greatly advance this research. One of the future prospects that emerged from the study is the expansion of the types of organic groups in the framework. Not limited to the organic groups seen in commercial phosphonate compounds, it is necessary to design molecular structures of bridged organic groups assuming all sorts of chemical approaches, including organic synthesis of phosphonate esters with desired bridged organic groups as well as addition of functional groups to phosphonate esters. I believe functional design using organic groups in the framework will be done with future orientation. In this research approach, reactivity can be controlled continuously through partial acid treatment of phosphonate esters. However, I do not think this is a universal method for all inorganic materials. Moreover, I have not reached the level of synthesis considering crystallinity of inorganic frameworks of mesoporous materials, and new ideas are necessary for its realization.

It is not certain that the concept of continuous reactivity control can be introduced to the side of inorganic species. In the future, it is necessary to develop synthesis methods for diverse mesoporous materials, being mindful not only of understanding the reactivity of inorganic materials during the early stages of reaction but also of how to control the reactivity of inorganic species that is taking place continuously in solution. Moreover, I hope to advance to a synthesis method that allows maximization of the crystalline property of inorganic frameworks. By doing so, the diversification of inorganic species to this point can be positioned as efforts toward "designing the pore environment." "Functional design derived from inorganic species" can be added to future design guidelines. Simple usages of mesoporous metal phosphonates are summarized in several papers. ${ }^{[26]-[29]}$ In the sense of future-oriented material design, metal phosphate and its metal oxides are predicted to show similar properties that derive from the property of metal species. Therefore, in the future, I wish to discuss possibilities for materials that act as alternatives in application development for non-silica-based oxide materials of which achievement of mesoporosity and powder synthesis technologies are currently not sufficiently established.

\section{Acknowledgement}

In conducting this research, I had pride of a synthesis researcher and the will to succeed despite insufficient funding. Therefore, I made sure my papers were published in illustrious international academic journals, and continued to generate steady research results, sometimes through collaboration with exterior research institutions. My efforts have come to fruition. Very recently, I was able to greatly advance this research, through the support of the Grant-inAid for Scientific Research (B), "Development of a precise synthesis technology for non-silica-based hybrid mesoporous materials by the molecular structure design (FY 2014-2016)" Japan Society for the Promotion of Science (JSPS KAKENHI Grant No. 26288110). I am grateful for this opportunity.

\section{References}

[1] T. Yanagisawa, T. Shimizu, K. Kuroda and C. Kato: The preparation of alkyltrimethylammonium-kanemite complexes and their conversion to microporous materials, Bull. Chem. Soc. Jpn., 63 (4), 988-992 (1990).

[2] M. E. Davis: Ordered porous materials for emerging applications, Nature, 417 (6891), 813-821 (2002).

[3] C. T. Kresge, M. E. Leonowicz, W. J. Roth, J. C. Vartuli and J. S. Beck: Ordered mesoporous molecular sieves synthesized by a liquid-crystal template mechanism, Nature, 359 (6397), 710-712 (1992).

[4] Q. Huo, D. I. Margolese, U. Ciesla, D. G. Demuth, P. Feng, T. E. Gier, P. Sieger, A. Firouzi, B. F. Chmelka, F. Schüth and G. D. Stucky: Organization of organic molecules with inorganic molecular species into nanocomposite biphase arrays, Chem. Mater., 6 (8), 1176-1191 (1994).

[5] T. Kimura: Surfactant-templated mesoporous aluminophosphate-based materials and the recent progress, Microporous Mesoporous Mater., 77 (2-3), 97-107 (2005).

[6] C. J. Brinker and G. W. Scherer: Sol-Gel Science: The Physics and Chemistry of Sol-Gel Processing, Academic Press, Boston (1990).

[7] F. Hoffmann, M. Cornelius, J. Morell and M. Fröba: Silicabased mesoporous organic-inorganic hybrid materials, Angew. Chem. Int. Ed. Engl., 45 (20), 3216-3251 (2006).

[8] S. Inagaki, S. Guan, T. Ohsuna and O. Terasaki: An ordered mesoporous organosilica hybrid material with a crystal-like wall structure, Nature, 416 (6878), 304-307 (2002).

[9] S. Fujita and S. Inagaki: Self-organization of organosilica solids with molecular-scale and mesoscale periodicities, Chem. Mater., 20 (3), 891-908 (2008).

[10] M. Waki, Y. Maegawa, K. Hara, Y. Goto, S. Shirai, Y. Yamada, N. Mizoshita, T. Tani, W. J. Chun, S. Muratsugu, M. Tada, A. Fukuoka and S. Inagaki: A solid chelating ligand: periodic mesoporous organosilica containing 2, 2'-bipyridine within the pore walls, J. Am. Chem. Soc., 136 (10), 4003-4011 (2014).

[11] Y. Lu, R. Ganguli, C. A. Drewien, M. T. Anderson, C. J. Brinker, W. Gong, Y. Guo, H. Soyez, B. Dunn, M. H. Huang and J. I. Zink: Continuous formation of supported cubic and hexagonal mesoporous films by sol-gel dip-coating, Nature, 389 (6649), 364-368 (1997).

[12] Y. Lu, H. Fan, A. Stump, T. L. Ward, T. Rieker and C. J. Brinker: Aerosol-assisted self-assembly of mesostructured spherical nanoparticles, Nature, 398 (6724), 223-226 (1999). 
[13] T. Kimura: Evaporation-induced self-assembly process controlled for obtaining highly ordered mesoporous materials with demanded morphologies, Chem. Rec., 16 (1), 445-457 (2016).

[14] H. Elhamzaoui, B. Jousseaume, H. Riague, T. Toupance, P. Dieudonné, C. Zakri, M. Maugey and H. Allouchi: Selfassembled tin-based bridged hybrid materials, J. Am. Chem. Soc., 126 (26), 8130-8131 (2004).

[15] T. Kimura: Synthesis of novel mesoporous aluminum organophosphonate by using organically bridged diphosphonic acid, Chem. Mater., 15 (20), 3742-3744 (2003).

[16] T. Kimura: Synthesis of mesostructured and mesoporous aluminum organophosphonates prepared by using diphosphonic acids with alkylene groups, Chem. Mater., 17 (2), 337-344 (2005).

[17] T. Kimura: Oligomeric surfactant and triblock copolymer syntheses of aluminum organophosphonates with highly ordered mesoporous structures, Chem. Mater., 17 (22), 5521-5528 (2005).

[18] T. Kimura and K. Kato: Simple removal of oligomeric surfactants and triblock copolymers from mesostructured precursors of ordered mesoporous aluminum organophosphonates, Microporous Mesoporous Mater., 101 (1-2), 207-213 (2007).

[19] T. Kimura and K. Kato: Synthesis of ordered mesoporous aluminum alkylenediphosphonates with integrated inorganic-organic hybrid frameworks, J. Mater. Chem., 17 (6), 559-566 (2007).

[20] T. Kimura: Advance in the synthetic strategy of mesoporous aluminum phosphonates, Phosphorus Letter, 91, 20-27 (2018) (in Japanese).

[21] B. Tian, X. Liu, B. Tu, C. Yu, J. Fan, L. Wang, S. Xie, G. D. Stucky and D. Zhao: Self-adjusted synthesis of ordered stable mesoporous minerals by acid-base pairs, Nature Mater., 2 (3), 159-163 (2003).

[22] T. Kimura: Molecular design of bisphosphonates to adjust their reactivity toward metal sources for the surfactantassisted synthesis of mesoporous films, Angew. Chem. Int. Ed., 56 (43), 13459-13463 (2017).

[23] T. Kimura, K. Kato and Y. Yamauchi: Temperaturecontrolled and aerosol-assisted synthesis of aluminum organophosphonate spherical particles with uniform mesopores, Chem. Commun., 33, 4938-4940 (2009).

[24] T. Kimura, N. Suzuki, P. Gupta and Y. Yamauchi: Effective mesopore tuning using aromatic compounds in the aerosolassisted system of aluminum organophosphonate spherical particles, Dalton Trans., 39 (21), 5139-5144 (2010).

[25] T. Kimura and Y. Yamauchi: General information to obtain spherical particles with ordered mesoporous structures, Chem. Asian J., 8 (1), 160-167 (2013).

[26] TY. Ma and ZY. Yuan: Metal phosphonate hybrid mesostructures: environmentally friendly multifunctional materials for clean energy and other applications, ChemSusChem, 4 (10), 1407-1419 (2011).

[27] YP. Zhu, TZ. Ren and ZY. Yuan: Mesoporous non-siliceous inorganic-organic hybrids: a promising platform for designing multifunctional materials, New J. Chem., 38 (5), 1905-1922 (2014).

[28] YP. Zhu, TY. Ma, YL. Liu, TZ. Ren and ZY. Yuan: Metal phosphonate hybrid materials: From densely layered to hierarchically nanoporous structures, Inorg. Chem. Front., 1 (5), 360-383 (2014).

[29] T. Kimura: A new family of nonsiliceous porous hybrids from bisphosphonates, J. Nanosci. Nanotechnol., 13 (4), 2461-2470 (2013).

\section{Author}

\section{Tatsuo KImURA}

Graduated from the Department of Applied Chemistry, School of Science and Engineering, Waseda University in March 1994. Completed courses at the Department of Applied Chemistry, Graduate School of Science and Engineering, Waseda University in March 1999; obtained doctorate (Engineering). Research Associate, Department of

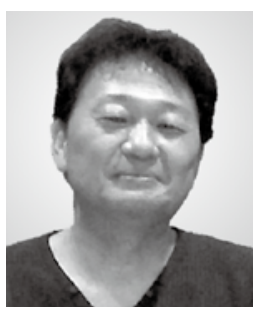
Applied Chemistry, School of Science and Engineering, Waseda University from April 1998. Joined the National Industrial Research Institute of Nagoya (currently, AIST Chubu) in October 2000; dispatched to the Nonferrous Metals Division and Metal Industries Division, Manufacturing Industries Bureau, the Ministry of Economy, Trade and industry (METI); and Group Leader, Materials for Chemical Transformation Group, Inorganic Functional Materials Research Institute, AIST from October 2016. Specialty is the inorganic materials chemistry. Engages mostly in researches on the precise synthesis of porous materials.

\section{Discussions with Reviewers}

\section{Overall}

Comment (Toshimi Shimizu and Motoyuki Akamatsu, AIST)

This paper describes, in scenario style, the details of the course by which synthesis of a non-silica-based hybrid mesoporous material, which was considered difficult, was achieved. The challenging strategies and tactics involve selection of new starting materials and utilization of unconventional synthesis routes. I think it is significant that you were able to extend the composition and structure of mesoporous materials as a result of this research. The paper demonstrates that the integration of cumulative elemental technologies is important, using tools of sub-nanometer scale chemical species such as organic and metal species. Hence, the paper is suitable for publication in Synthesiology.

\section{Synthesis and integration of elemental technologies Comment \& Question (Toshimi Shimizu)}

The author uses Fig. 3 to show individual items (1) to (6) as elemental technologies for the synthesis research for mesoporous materials, and emphasizes that their integration is essential in composition design control of advanced mesoporous materials. However, looking at the subchapter titles of the first draft of this paper, rather than explanations of elemental technologies from (1) to (6), you provide explanations of new chemical materials and synthesis routes from silica to organosilica, alumina and titania, and then non-silica-based hybrid mesoporous materials. In other words, there is no specific explanation about the integration of elemental technologies. To deepen the readers' understanding, I think you should clearly state what correlation exists between newly developed chemical materials and synthesis routes and the elemental technologies (1) to (6), as well as specific contents about the integration of elemental technologies. Also, here is a question: Is the route newly developed by the author (Fig. 4 and Fig. 5) neither the liquid crystal template route shown in Fig. 3 nor the concerted organization route?

Answer (Tatsuo Kimura)

In the process of synthesizing mesoporous silica shown in 
Fig. 1, the elemental technologies that must be considered can be categorized into (1) to (6). Excluding elemental technology (6), it is correct in understanding that mesoporous silica is synthesized by their integration. The importance becomes more apparent in materials like non-silica-based materials in which obtaining mesoporosity is difficult. The mesoporous materials cannot be synthesized just by improving individual elemental technologies. Therefore, particularly important technology is how to control bond formation of inorganic species in solution, as stated in elemental technology (1). This is explained in the section about the difficulties of synthesizing non-silica-based mesoporous materials. Moreover, in elemental technology (5) that seems to have low relationship with others, the results are affected by the integrity of the periodic structure (high symmetry) and the degree of condensation of the silicate framework in the mesoporous silica precursor. As a result, it is reported that the mesoporous structure disintegrates during the process of removing amphiphilic organic molecules.

The precursors of mesoporous materials is invariably formed by either the liquid crystal template route or the concerted organization route. In this research a new formation route was not developed. Therefore, I corrected Fig. 4 and Fig. 5 so the details of the synthesis route would correspond to the numbering of elemental technologies. In this research, new composition design technology was proposed and demonstrated using the reaction of organic bridged phosphonate compounds and metal sources to achieve non-silica-based inorganic-organic composites with the ingredients that surround the assembly of amphiphilic organic molecules.

\section{Comment (Motoyuki Akamatsu)}

In the first two paragraphs of Chapter 2, you describe the silica-based synthesis route and that synthesis occurs through a concerted organization route. Based on this, you identify six elemental technologies, and your claim is that these six elemental technologies must be integrated for non-silica-based materials. Then you describe the development of non-silicabased mesoporous materials in Chapter 3 in which you state the selection of materials in Subchapter 3.0 and reactivity control in Subchapter 3.2. However, non-specialist readers cannot easily understand which of the six elemental technologies corresponds to which part of the description. Can you make the corresponding relation easier to understand? I think it will make things clear if explanations of the elemental technologies follow the subchapters of Chapter 3.

\section{Answer (Tatsuo Kimura)}

I thought I clearly showed the important elemental technologies in the figure for formation mechanism of Fig. 4 (silica-based hybrid materials) and Fig. 5 (non-silica-based hybrid materials). However, it seems that it was difficult to understand for non-specialist readers, so I decided to use the numbering of the six elemental technologies.

\section{Research strategy}

\section{Comment \& Question (Motoyuki Akamatsu)}

In Paragraph 5, Chapter 1, you explain that while a study of hybrid mesoporous materials by silica-based materials is R\&D that "specializes on functional design," this research (= non-silica-based hybrid mesoporous materials) took a research strategy (= research policy?) of "designing the pore environment." What is the essential difference between the two? While silicabased materials followed a goal-oriented approach (of developing a material to realize a certain function), why didn't you take such a goal-oriented approach here? Please explain your views behind this.

\section{Answer (Tatsuo Kimura)}

The essential difference of the research strategies of "specializing in functional design" and "designing the pore environment" is as follows.

Silica-based mesoporous material is made to express functions (acidity, oxidizing function, etc.) that are not expressed with silica alone by introducing different element species into the silicate framework, and at times organic groups are incorporated into the silica framework as in mesoporous organosilica. In any case, design is specific such as for catalyst function or photoresponsivity, and components that are necessary for functional expression (goal oriented) are introduced. However, since there is no option other than using the pore environment provided by the final product, the design of the environment inside the pore is essential for efficient functional expression.

In this research, I discuss the difficulty of synthesis research for hybrid mesoporous materials and the level I have achieved so far. In other words, the discussion is about the methodology for designing the pore environment. Ultimately, if I utilize the findings necessary for functional design that had been clarified for silica-based materials, I believe I can design a nanoscale chemical reaction field where functions can be expressed efficiently.

For the question why I did not take a goal-oriented approach for non-silica-based hybrid mesoporous materials that is the target of this research, that is because I have not reached the stage in which I can freely synthesize with a goal in mind. Even for a silica-based mesoporous material, it cannot be synthesized on demand. Because there are overwhelming number of reports and the selection range has expanded, it merely looks like there are lots of accomplishments in "specializing on functional design" that is actually the next phase of research. In non-silicabased materials, as described in this paper, the reactivity of raw materials is fast, and an enormous amount of experimental samples are needed to synthesize just one type of mesoporous material with a certain inorganic composition. Therefore, I think there is great significance that I opened a path toward diversification of non-silica-based hybrid mesoporous materials through an approach of continuous control of reactivity of the raw materials, as shown in this study.

\section{New properties of materials and expanded usage Comment (Toshimi Shimizu)}

By extending the composition of mesoporous materials from the current silica to organosilica, or from silica to alumina and then to titania, and from organosilica to non-silica hybrids, I can expect the expansion of structure, function, and property value of mesoporous materials. To strengthen the significance of this paper, how about adding specific new properties and advantages I can expect through the development of a universal synthesis method for non-silica-based hybrid mesoporous materials? Also, if possible, I think it will help the readers understand the research objective if you add the potential and expectations on how such development will contribute to industrial and social demands. There is a section at the bottom of Fig. 1 that points to this. However, it is organized by items "silica and alumina," "transition metal oxides," "carbon," "metals," and "hybrid materials," and they do not correspond to the terminology used in this paper like "non-silica-based" or "non-silica-based hybrid."

\section{Answer (Tatsuo Kimura)}

For the point that you indicated, I added a text to Paragraph 3, Chapter 1 of the final draft. Also, for the section at the bottom of Fig. 1, I added a simple explanation to Paragraph 3, Chapter 1.

\section{Comment \& Question (Motoyuki Akamatsu)}

In Chapter 1, you write, "the primary research strategy of this research is the design of pore environment," and after that you write, "I set the goal as the design of hydrophilic surface structure." Does this mean the specific goal of "designing the pore environment" is the "designing of the hydrophilic surface 
structure"? Also, I understood that you set such a goal because, with silica-based materials, you can only do certain application development because they are hydrophobic, but you expect that the usage will expand if they can be made hydrophilic. Can you explain to which fields the usage can expand if you can make it hydrophilic compared to the silica usage shown in Fig. 1?

\section{Answer (Tatsuo Kimura)}

As you indicate, I started efforts on "designing the hydrophilic surface structure" for which there were hardly any reports, as my specific goal of "designing the pore environment." Silica-based and all other mesoporous materials have hydrophobic pore environment, and the pore environment becomes even more hydrophobic in application development using organic groups. Therefore, as the first goal, I attempted to create hydrophilic nanospace directly opposite. Therefore, I assumed usages as catalyst carriers and adsorbents that are major uses of silica-based mesoporous materials as shown in Fig. 1. If I can achieve hydrophilic surfaces, I expect, for example, application development as a chemical reaction field to greatly increase reaction efficiency of chemical substances including hydrophilic regions.

\section{Reactivity control of inorganic species Question (Toshimi Shimizu)}

Among elemental technologies, if you change from silicabased to non-silica-based, I think it would be very important what strategies and tactics are employed for (1) reactivity control of inorganic species. To control the reactivity of inorganic species, "a $\mathrm{Si}-\mathrm{O}-\mathrm{Si}$ bond is formed by inserting an organic cross-link region between the silica species, or a P-O-M bond is formed using the interaction of phosphonic acid and metal species by inserting the organic cross-link region between phosphonic acids. If the precursor of porous materials can be formed by self-assembly, the key will be how to extract the core parts (by solvent extraction, low temperature firing, dissolution extraction, etc.)." Is my understanding correct?

\section{Answer (Tatsuo Kimura)}

For the understanding of reactivity control of inorganic species, I shall add some explanation particularly to the part you indicated. In the final product, a starting material to which an organically bridged part has been inserted to create an inorganicorganic composite framework is used. However, this changes the reactivity of the raw material but does not directly lead to control.

For example, in the case of silica, the initial reactivity of the material changes by whether chlorosilane $(\mathrm{Si}-\mathrm{Cl})$ is used or alkoxylsilane (Si-OR) is used. The formation speed of the Si-OSi bond changes, not only by hydrolysis of initial reaction, but also by the $\mathrm{pH}$ at which the precursor solution is prepared. As the reaction progresses, the hydrophilic regions of the amphiphilic organic molecules and dissolved silicate species interact, a silicate framework is formed as further bond formation takes place as self-assembly progresses, and the precursor of mesoporous silica is obtained. If the reaction of silicate species goes too far, the charge density of the silicate framework decreases, the interacting amphiphilic organic molecules fall off, and only mesoporous silica precursors with lowered integrity of periodic structure are obtained.

In non-silica-based oxides with higher reactivity, a precipitate consisting of only inorganic species is formed. Thus obtaining even the precursor of mesoporous materials becomes impossible. However, in the case of achieving mesoporosity of metal phosphates, phosphates are used as the phosphorus source in almost all cases, and the initial reactivity changes by whether chloride (M-Cl) or alkoxides (M-OR) are used as metal sources. In this case, when phosphates are coexisting at a certain ratio, the metal sources and phosphates react first (P-O-M bond forms), and if the reaction is relatively slow, the following reaction will be relatively mild. In a case in which formation of a P-O-M bond is fast, as in the non-silica-base, it is necessary to inhibit the reactivity in solution, and measures must be taken to prepare an appropriate non-aqueous precursor solution.

Considering the above, even in synthesis using phosphonate compounds instead of phosphates, as explained in the paper, the insertion of organically bridged regions must be done at the same time as the functional design (to realize inorganic-organic composites). Since electron density of phosphorus atoms changes according to the type of organic groups, the initial reactivity of the raw material changes. For the control, this research proposes a new method of controlling reactivity using a starting material in which the ester region (P-OR) and hydroxyl groups $(\mathrm{P}-\mathrm{OH})$ coexist in the same molecular structure.

\section{Final goal \\ Comment \& Question (Motoyuki Akamatsu)}

In the first paragraph of Chapter 3, you write "I set the final goal or 'point of achievement in synthesis research' as opening the way to advanced material design," but does this mean you set as your goal the development of universal synthesis technology? In the latter half of this paragraph, you write about the starting material, but is this the starting material that leads to universal synthesis technology? Also, is this the organically bridged phosphonates that you introduce in Subchapter 3.1? In Discussion 2 , in relation to my question, the logical progression of this part is unclear, so please clarify.

\section{Answer (Tatsuo Kimura)}

As I wrote immediately after the part you indicated. in the research field of mesoporous materials, the significance of synthesis research of non-silica-based hybrid mesoporous material is opening the way to advanced materials design. As a result, if it is proven that the pore environment can be controlled arbitrarily, advanced application development can be started as the next research phase. There isn't much meaning in doing usage tests to match the developed material. Therefore, I focus on synthesis research, and aim to provide a series of mesoporous materials with various pore environments. For logical progression, I added corrections in the indicated areas. 